\title{
Photonic metal oxide gas sensors: low temperature ozone sensing by cyclic optical excitation of $\ln _{2} \mathrm{O}_{3}$
}

\author{
Daniel Meixner ${ }^{1}$, Claus-Dieter Kohl'1, Thorsten Wagner ${ }^{2}$ \\ 1 Justus Liebig University Giessen, Leihgesterner Weg 217, D-35392 Giessen, Germany \\ 2 University of Paderborn, Warburger Str. 100, D-33098 Paderborn, Germany \\ Thorsten.Wagner@upb.de
}

\begin{abstract}
:
Enhancing and activating reactivity of metal oxides to gases by optical excitation is already a well investigated field of heterogeneous catalysis. Observed effects as e.g. the already mentioned activity enhancement or increasing selectivity are also of high interest in the field of resistive semiconducting gas sensors. Most of the already investigated systems are nanostructured. However, even though many positive influences on the sensing performance were observed, there are only a very few systematic investigations related to the basic effects. In the current work we therefore like to present a systematic study on cyclic illumination of nanostructured indium oxide $\left(\mathrm{In}_{2} \mathrm{O}_{3}\right)$ for gas sensing applications. Indium oxide is a suitable model material since it can be used to detect oxidizing gases even at room temperature. Therefore the influence of illumination can be separated from thermal activation. We present results on low temperature ozone sensing and generation of multiple signals by cyclic illumination of ordered nanoporous $\ln _{2} \mathrm{O}_{3}$ utilizing blue LEDs.
\end{abstract}

Key words: optical excitation, semiconducting gas sensor, indium oxide, cyclic illumination, ozone sensing

\section{Introduction}

Indium oxide is a promising candidate for optically activated low temperature $\left(<100{ }^{\circ} \mathrm{C}\right)$ resistive sensing of oxidizing gases like e.g. ozone or nitrous oxides. It offers an inherent selectivity to oxidizing gases [1]. The low operating temperatures $\left(<100{ }^{\circ} \mathrm{C}\right)$ correspond to a strongly reduced power consumption compared to regular semiconducting sensors operated at a few hundred ${ }^{\circ} \mathrm{C}$.

However, without thermal activation of the electronic and chemical processes sensor kinetics is typically slow. Especially desorption of adsorbates like $\mathrm{NO}_{2}$ is suppressed causing long regeneration times [2].

As an alternative optical activation by light in the UV/vis range was shown to affect kinetics of different sensing materials $[3,4]$. In case of nanostructured $\mathrm{In}_{2} \mathrm{O}_{3}$ continuous illumination significantly speeds up sensor reaction as well as regeneration under exposure of ozone or $\mathrm{NO}_{2}[5,6]$. The observed effects can be explained by means of a new sensing model which considers oxidation and (photo) reduction responsible for the sensing reaction [7].
For specific combinations of material and structuring continuous illumination of sensing layers was shown to correspond to constant temperature operation mode [8]. The impact of illumination seems to be strongest for nanostructured materials. In case of compact, non-nanostructured sensing layers cyclic illumination was shown to be advantageous [9]. This can be explained by considering a limited penetration depth of the optical activation. In contrast to thermal activation which affects the whole sensing layer optical activation only penetrates a thin, surface-near region (e.g. ca. $10 \mathrm{~nm}$ for $\mathrm{In}_{2} \mathrm{O}_{3}$ [10]). Therefore the structure of the sensing layer as well as of the sensing material strongly affects the sensor behaviour under illumination [11].

However, as for the best of authors knowledge there are so far no studies systematically investigating cyclic illumination. In the following we present first results on studying the influence of cyclic illumination on the sensor kinetic and response as well as the utilization for the generation of multiple signals with the aim of selectivity enhancement. This is already a well-established method in the field of thermocyclic operation e.g. [12]. 


\section{Experimental}

\section{Sensor preparation}

Nanostructured (ordered mesoporous) $\quad \ln _{2} \mathrm{O}_{3}$ was synthesized by structure replication (nanocasting) of a mesoporous KIT-6 silica matrix. Particle size control was achieved utilizing variations of gas atmosphere during precursor conversion. The resulting particles exhibit pores with a diameter of ca. $5 \mathrm{~nm}$ and a size of $170 \mathrm{~nm}$. Details regarding the synthesis procedure and characterization can be found elsewhere [13].

Sensing layers were prepared by drop-coating aqueous dispersions ( $25 \mathrm{mg}$ in $1 \mathrm{~mL} \mathrm{H}_{2} \mathrm{O}$ dest.) onto commercially available sensor substrates (UST $\mathrm{GmbH}$, alumina based, $3 \times 3 \mathrm{~mm}$ with interdigitated electrodes and Pt10 heater). Layer thickness was estimated by SEM to ca. $10 \mu \mathrm{m}$. Prior first measurement sensing layers are heated to $175{ }^{\circ} \mathrm{C}$ in air for 24 hours to remove residual water from the drop coating process.

\section{Resistance measurement}

The sensing layer resistance was measured in potentiostatic mode at a voltage of $0.5 \mathrm{~V}$ utilizing custom build electronics.

For illumination a blue LED with a wavelength peak maximum at $466 \mathrm{~nm}$ was used. The light source was mounted at a distance of $3 \mathrm{~mm}$ to the sensing layer in a custom PTFE housing. The LED was driven by a precision current source controlled by a $\mu$-controller allowing different operation modes. In the continuous illumination mode the LED was driven by a constant current of $26 \mathrm{~mA}$. In the cyclic illumination mode symmetric square shaped current pulses with a gradient of $\pm 26 / 0.01 \mathrm{~mA} / \mathrm{s}$ at the switching points and an amplitude of 26 $\mathrm{mA}$ were used. The periodicity of the signal was 20 seconds, i.e. the sensors were illuminated for 10 seconds followed by a dark period of 10 seconds.

\section{Gas mixing}

A custom build gas mixing equipment based on mass flow controllers was utilized to supply the desired ozone concentrations in synthetic air. The ozone was generated utilizing a commercially available ozone generator (UVP Modell SOG-1) and controlled with an ozone monitor (2B Technologies Modell 202). Concentration was varied by controlling the light intensity of the generator.

\section{Results and discussion}

In a first experiment the sensor reaction to cyclic illumination in air was tested. As can be seen in Fig. 1 the illumination profile is causing a sawtooth shaped resistance change. During illumination period a resistance decrease can be observed which is most likely due to electronic activation and photo-desorption increasing the number of free charge carriers. After turning off the illumination relaxation processes and adsorption of gas species then lead to an increase of the resistance again. After a few cycles a dynamic equilibrium is reached. As can be seen the cycle shape is not symmetric which suggests that it contains information about the kinetics of the underlying processes.

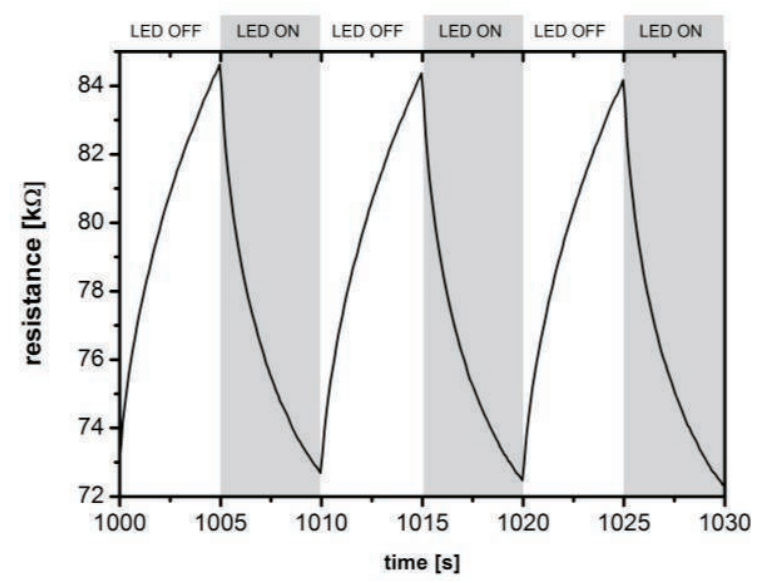

Fig. 1 Resistance profile of mesoporous $\ln _{2} \mathrm{O}_{3}$ layer caused by cyclic illumination $(460 \mathrm{~nm})$ in air at room temperature.

To evaluate the influence of the cyclic illumination on gas reaction the sensor response to a $100 \mathrm{ppb}$ ozone exposure of 20 minutes was compared to different illumination scenarios (flow rate $200 \mathrm{ml} / \mathrm{min}$, relative humidity $20 \%$ ).
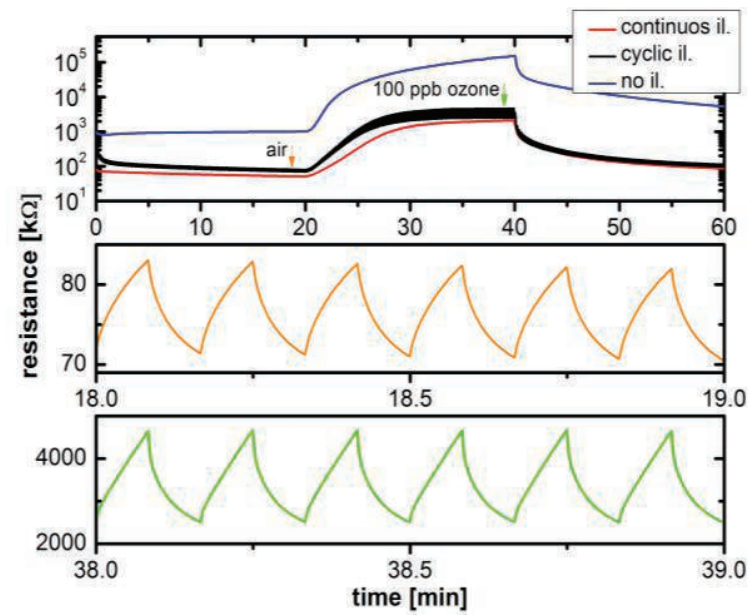

Fig. 2 Comparison of sensor reaction of $\operatorname{In}_{2} \mathrm{O}_{3}$ to 100 ppb ozone exposure at continuous (red), cyclic (black) and without illumination (blue) with blue light $(460 \mathrm{~nm})$. 
Results show (Fig.2) that the mean value of the resistance in cyclic illumination mode is located between the continuously illuminated sensor and the sensor operated without illumination.

Table 1 summarizes some data regarding sensor response (defined here as $\mathrm{R} / \mathrm{R}_{0}$ where $\mathrm{R}$ and $R_{0}$ are the Ohmic resistances in the presence and absence of the test gases, respectively) and kinetics. The highest response values are achieved in the nonilluminated case. Consistent with results obtained in former works [6] the t9o times for reaction and regeneration are orders of magnitude higher than in the continuously illuminated case. The most remarkable result, however, is the improved kinetics of the cyclic illuminated sensor. Especially the regeneration time is only half the value than for the continuously illuminated sensor.

Tab. 1: Results of the comparative ozone measurement

\begin{tabular}{|c|c|c|c|c|}
\hline illumin. & $\begin{array}{c}\text { resp. } \\
{\left[\mathrm{R} / \mathrm{R}_{0}\right]}\end{array}$ & $\begin{array}{c}\text { t90,reaction } \\
{[\mathrm{s}]}\end{array}$ & $\begin{array}{c}\text { t90,regen. } \\
{[\mathrm{s}]}\end{array}$ & $\begin{array}{c}\text { t95,regen. } \\
{[\mathbf{s}]}\end{array}$ \\
\hline no & 150 & $>>1000$ & 350 & 770 \\
\hline cyclic & 55 & 700 & 100 & 250 \\
\hline cont. & 40 & 900 & 230 & 480 \\
\hline
\end{tabular}

Besides the response and kinetic also the shape of the response cycle changes during ozone exposure. The amplitude of the sawtooth profile increases from $15 \mathrm{k} \Omega$ (without ozone) to $1300 \mathrm{k} \Omega$ in ozone environment. Just as noticeable is the change in shape of the rising and falling edge during on- and off-time of the LED, respectively. This suggests that further data analysis utilizing e.g. principal component analysis (PCA) or similar methods will allow for selectivity enhancement.

Since humidity is one of the most common interfering gases further measurements with humidity variations under cyclic illumination conditions were performed. As typical for room temperature operation of semiconducting sensing materials the mean value of the sensor resistance showed some unpredictable behavior (see Fig. 3). The absolute resistance value cannot be correlated to the relative humidity level since the signal seems to be super-positioned by some long term drift effects. However, detailed analysis of the resistance cycles reveals differences in amplitude and shape.

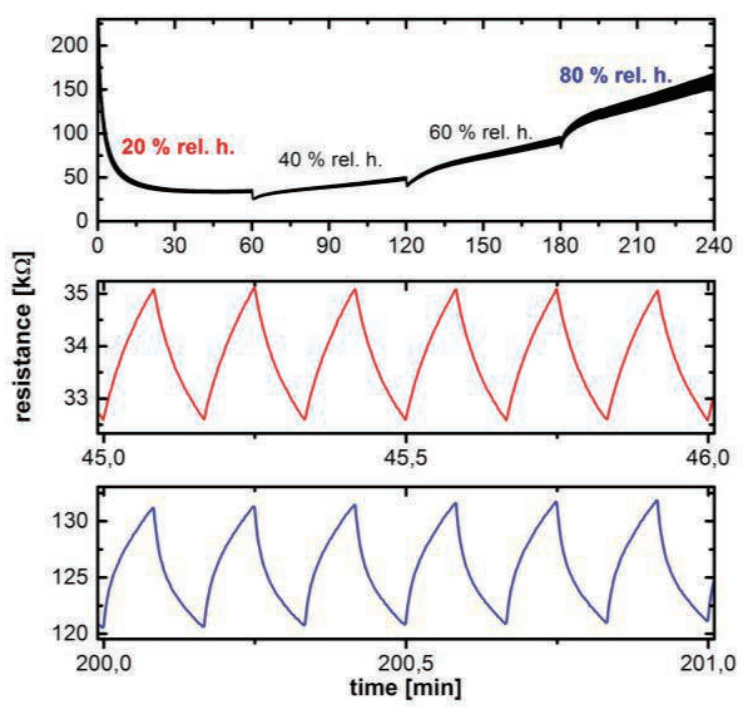

Fig. 3 Resistance change of $\mathrm{In}_{2} \mathrm{O}_{3}$ at cyclic illumination caused by changes in relative humidity (top); detailed view of cycles at $20 \%$ (red) and $80 \%$ (blue) relative humidity, respectively.

At a relative humidity of $20 \%$ the amplitude is about $3 \mathrm{k} \Omega$ and changes to $10 \mathrm{k} \Omega$ for $80 \%$. Defining the sensor response as

response $=\left(1 / R_{\min , \text { cylce }}-1 / R_{\max , \text { cycle }}\right)^{-1}$

enables for clear separation of the different humidity levels (see Fig. 4).

Future experiments will need to cover mixedgas situations, e.g. change in humidity during ozone exposure, as well. Since the curvature of the rising edge during humidity variations differs from the curvature change during ozone exposure chances are good that a separation still will be possible.

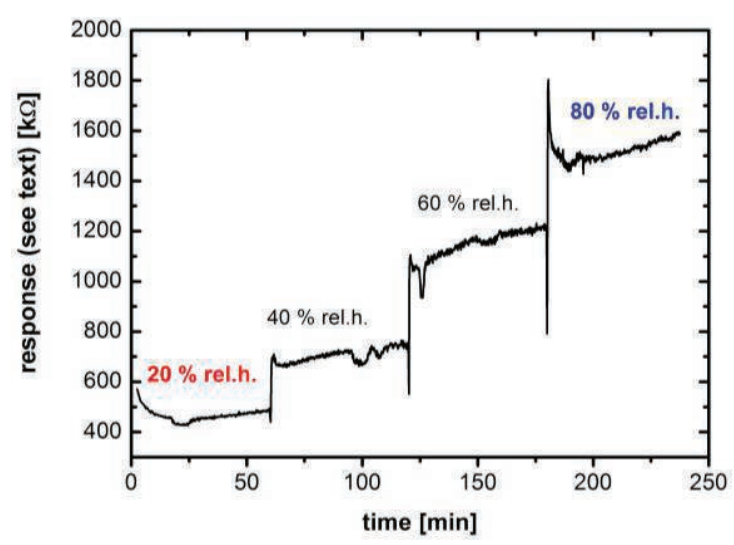

Fig. 4 Sensor response to different humidity levels.

At first the sensor was illuminated continuously in the second run the sensor was operated in the above described cyclic illumination mode and at last the sensor response was measured without illumination. Between measurements 
the sensor was regenerated for 10 hours in air without illumination.

\section{Conclusion}

Nanoporous $\ln _{2} \mathrm{O}_{3}$ is highly sensitive to oxidizing gases (e.g. ozone) at room temperature but with poor kinetics. The presented measurements shows that especially cyclic illumination of the sensing layer is a proper tool for improving the response and recovery times significantly.

However, details about the underlying mechanisms are still under investigation. A deeper understanding will enable further improvements of the operation mode as well as allowing for optimization of structural parameters of the sensing material and the sensing layers

The present study also showed that cyclic illumination creates characteristic resistance profiles for different gases suggesting that selectivity enhancement by multiple signal evaluation techniques will be possible.

\section{References}

[1] W.-Y. Chung, Y.-S. Lee, D.-D. Lee, Indium oxide thin film sensors for ozone monitoring produced by a sol-gel method, Journal of Materials Science Letters 22 (2003) 907-909.

[2] M. Law, H. Kind, B. Messer, F. Kim, P. Yang, Photochemical Sensing of $\mathrm{NO}_{2}$ with $\mathrm{SnO}_{2}$ Nanoribbon Nanosensors at Room Temperature, Angewandte Chemie 114 (2002) 2511-2514.

[3] S. Bianchi, E. Comini, M. Ferroni, G. Faglia, A. Vomiero, G. Sberveglieri, Indium oxide quasimonodimensional low temperature gas sensor: Eurosensors XIX - Eurosensors XIX - The 19th European Conference on Solid-State Transducers, Sensors and Actuators B: Chemical 118 (2006) 204-207.

[4] E. Comini, G. Faglia, G. Sberveglieri, UV light activation of tin oxide thin films for $\mathrm{NO}_{2}$ sensing at low temperatures: Selected Papers from Eurosensors XIV, Sensors and Actuators B: Chemical 78 (2001) 73-77.

[5] T. Wagner, C.-D. Kohl, C. Malagù, N. Donato, M. Latino, G. Neri, M. Tiemann, UV lightenhanced $\mathrm{NO}_{2}$ sensing by mesoporous $\ln _{2} \mathrm{O}_{3}$ : Interpretation of results by a new sensing model, Sensors and Actuators B: Chemical 187 (2013) 488-494.

[6] T. Wagner, J. Hennemann, C.-D. Kohl, M. Tiemann, Photocatalytic ozone sensor based on mesoporous indium oxide: Influence of the relative humidity on the sensing performance: Special Section: Proceedings of 7 th International Workshop on Semiconductor Gas Sensors, Thin Solid Films 520 (2011) 918-921.

[7] C.-D. Kohl, T. Wagner (Eds.), Gas sensing fundamentals. Springer Series on Chemical Sensors and Biosensors (2014) ISBN 978-3642-54519-1.
[8] J.D. Prades, R. Jimenez-Diaz, F. HernandezRamirez, S. Barth, A. Cirera, A. RomanoRodriguez, S. Mathur, J.R. Morante, Equivalence between thermal and room temperature UV light-modulated responses of gas sensors based on individual $\mathrm{SnO}_{2}$ nanowires, Sensors and Actuators B: Chemical 140 (2009) 337-341.

[9] C.Y. Wang, V. Cimalla, T. Kups, C. Rohlig, T. Stauden, O. Ambacher, M. Kunzer, T. Passow, W. Schirmacher, W. Pletschen, K. Kohler, J. Wagner, Integration of $\ln _{2} \mathrm{O}_{3}$ nanoparticle based ozone sensors with GalnN/GaN light emitting diodes, Applied Physics Letters 91 (2007) 103509-3.

[10] M. Bender, N. Katsarakis, E. Gagaoudakis, E. Hourdakis, E. Douloufakis, V. Cimalla, G. Kiriakidis, Dependence of the photoreduction and oxidation behavior of indium oxide films on substrate temperature and film thickness, $J$. Appl. Phys. 90 (2001) 5382-5387.

[11] D. Klaus, D. Klawinski, S. Amrehn, M. Tiemann, T. Wagner, Light-Activated Resistive Ozone Sensing at Room Temperature Utilizing Nanoporous $\ln _{2} \mathrm{O}_{3}$ Particles: Influence of Particle Size, Sensors and Actuators B: Chemical (2014).

[12] A. Gramm, A. Schütze, High performance solvent vapor identification with a two sensor array using temperature cycling and pattern classification, Selected Papers from Eurosensors XVI, Sensors and Actuators B: Chemical 95 (2003) 58-65.

[13] D. Klaus, S. Amrehn, M. Tiemann, T. Wagner, One-step synthesis of multi-modal pore systems in mesoporous $\ln _{2} \mathrm{O}_{3}$ : A detailed study, Microporous and Mesoporous Materials 188 (2014) 133-139. 